九州大学学術情報リポジトリ

Kyushu University Institutional Repository

\title{
Duality in Dynamic Fuzzy Systems
}

Yoshida, Yuji

Faculty of Economics and Business Administration, Kitakyushu University

http://hdl. handle. net/2324/3194

出版情報 : RIFIS Technical Report. 104, 1995-02. Research Institute of Fundamental Information Science, Kyushu University

バージョン：

権利関係 : 


\title{
RIFIS Technical Report
}

Duality in Dynamic Fuzzy Systems

\author{
Y. Yoshida
}

February ,1995

Research Institute of Fundamental Information Science

Kyushu University 33

Fukuoka 812, Japan 


\title{
DUALITY IN DYNAMIC FUZZY SYSTEMS
}

\author{
Yuji YOSHIDA \\ Faculty of Economics and Business Administration, Kitakyushu University, \\ 4-2-1 Kitagata, Kokuraminami, Kitakyushu 802, Japan
}

\begin{abstract}
This paper shows the resolvent equation, the maximum principle and the co-balayage theorem for a dynamic fuzzy system. We define a dual system for the dynamic fuzzy system, and gives a duality for Snell's optimal stopping problem by the dual system.

Keyword: Duality, dynamic fuzzy system, optimal stopping problem, balayage theorem, resolvent equation, maximum principle.
\end{abstract}

\section{Introduction}

In [4], we defined a dynamic fuzzy system using a fuzzy relation and gave limit theorems for the transition of fuzzy states of the system under the contractive properties of the fuzzy relation. Next, in $[14,15]$, we discussed potential theory of the system under the contractive and nonexpansive properties. Further, in Yoshida [11], we developed fundamental theory of the dynamic fuzzy system as a Markov chain with transition possibility measures. This paper analyses dual properties for the system in [11], and introduces a dual system of the dynamic fuzzy system as a dual Markov chain with transition possibility measures.

In Sections 2 and 3, we define various fuzzy transition operators and $P$-excessive possibility measures. For the dynamic fuzzy system, we show the resolvent equation, the maximum principle and the co-balayage theorem, which are well-known in Markov potential theory. In Section 4, we define a dual dynamic fuzzy system, on the basis of the results.

In this paper, we deal with Snell's optimal stopping problem for the dynamic fuzzy system (see [11, Section 4]), and discuss a duality for the problem. We give a dual representation by the dual dynamic fuzzy system. In Section 5 , we also give a onedimensional numerical example to illustrate our idea.

\section{Dynamic fuzzy systems}

We use the notations of dynamic fuzzy systems introduced by [11]. Let $S$ be a metric space. We write a fuzzy set on $S$ by its membership function $\tilde{s}: S \mapsto[0,1]$ and an ordinary set $A(\subset S)$ by its indicator function $1_{A}: S \mapsto\{0,1\}$. Refer to Zadeh [16] and Novàk [6] 
for the theory of fuzzy sets. We define operations $\Lambda$ and $\vee$ for fuzzy sets as follows : Let $\Gamma$ be an index set. For a family of fuzzy sets $\left\{\tilde{s}_{n}\right\}_{n \in \Gamma}$ on $S$, we put

$$
\bigwedge_{n \in \Gamma} \tilde{s}_{n}(x):=\inf _{n \in \Gamma} \tilde{s}_{n}(x) \text { and } \bigvee_{n \in \Gamma} \tilde{s}_{n}(x):=\sup _{n \in \Gamma} \tilde{s}_{n}(x), \quad x \in S
$$

For a fuzzy set $\tilde{s}$ on $S$, its $\alpha$-cut $\tilde{s}_{\alpha}$ is defined by

$$
\tilde{s}_{\alpha}:=\{x \in S \mid \tilde{s}(x) \geq \alpha\}(\alpha \in(0,1]) \quad \text { and } \quad \tilde{s}_{0}:=\operatorname{cl}\{x \in S \mid \tilde{s}(x)>0\},
$$

where cl denotes the closure of a set. $\mathcal{E}(S)$ denotes the set of all countable unions of closed subsets of $S$, so called $F_{\sigma}$-sets (see [5] and [11]). $\mathcal{F}(S)$ denotes the set of all fuzzy sets on $S$ satisfying the following conditions (F.i) and (F.ii) :

(F.i) $\tilde{s}_{\alpha} \in \mathcal{E}(S)$ for $\alpha \in[0,1]$;

(F.ii) $\bigcap_{\alpha^{\prime}<\alpha} \tilde{s}_{\alpha^{\prime}}=\tilde{s}_{\alpha} \quad$ for $\alpha \in(0,1]$.

Then, we define

$\mathcal{G}(S):=\left\{\right.$ fuzzy sets $\tilde{s}$ on $S \mid$ there exists a sequence $\left\{\tilde{s}_{n}\right\}_{n=0}^{\infty} \subset \mathcal{F}(S)$ such that $\left.\bigvee_{n \geq 0} \tilde{s}_{n}=\tilde{s}\right\}$.

Let a time space $\mathbf{N}:=\{0,1,2,3, \cdots\}$. Let a state space $E$ be a finite-dimensional Euclidean space. We put a path space by $\Omega:=\prod_{k=0}^{\infty} E$ and we put a sample path $\omega=(\omega(0), \omega(1), \omega(2), \cdots) \in \Omega$. Define a map $X_{n}(\omega):=\omega(n)$ and a shift $\theta_{n}(\omega):=$ $(\omega(n), \omega(n+1), \omega(n+2), \cdots)$ for $n \in \mathbf{N}$ and $\omega=(\omega(0), \omega(1), \omega(2), \cdots) \in \Omega$. We put $\sigma$ fields by $\mathcal{M}_{n}:=\sigma\left(X_{0}, X_{1}, \cdots, X_{n}\right)^{1}$ for $n \in \mathbf{N}$ and $\mathcal{M}:=\sigma\left(\bigcup_{n \in \mathrm{N}} \mathcal{M}_{n}\right)^{2}$. In this paper, we call $X:=\left\{X_{n}\right\}_{n \in \mathrm{N}}$ a dynamic fuzzy system. The law of the transition is defined as follows. Let $\tilde{q}$ be time-invariant upper semicontinuous fuzzy relations on $E \times E$ satisfying the following normality condition :

$$
\sup _{x \in E} \tilde{q}(x, y)=1(y \in E) \quad \text { and } \quad \sup _{y \in E} \tilde{q}(x, y)=1(x \in E) .
$$

Then $\tilde{q}$ means a transition fuzzy relation. Define $\tilde{P}(\Lambda):=\sup _{\omega \in \Lambda} \Lambda_{n=0}^{\infty} \tilde{q}\left(X_{n}(\omega), X_{n+1}(\omega)\right)$ for $\Lambda \in \mathcal{M}$. We define a fuzzy expectation by the possibility measure $\tilde{P}$ : For an initial state $x \in E$,

$$
E_{x}(h):=f_{\{\omega \in \Omega: \omega(0)=x\}} h(\omega) \mathrm{d} \tilde{P}(\omega)=\sup _{\omega \in \Omega: \omega(0)=x} h(\omega) \wedge \bigwedge_{n=0}^{\infty} \tilde{q}\left(X_{n} \omega, X_{n+1} \omega\right)
$$

for all $\mathcal{M}$-measurable fuzzy sets $h \in \mathcal{F}(\Omega)$, where $f \mathrm{~d} \tilde{P}$ denotes Sugeno integral (Sugeno [10]).

\footnotetext{
${ }^{1}$ It denotes the smallest $\sigma$-field on $\Omega$ relative to which $X_{0}, X_{1}, \cdots, X_{n}$ are measurable.

${ }^{2}$ It denotes the smallest $\sigma$-field generated by $\bigcup_{n \in \mathbf{N}} \mathcal{M}_{n}$.
} 
Let $\mathcal{E}:=\left\{A \mid A \in \mathcal{E}(E)\right.$ and $\left.A^{c} \in \mathcal{E}(E)\right\}$, where $A^{c}:=E \backslash A$. We call a map $\tau: \Omega \mapsto$ $\mathbf{N} \cup\{\infty\}$ an $\mathcal{E}$-stopping time if

$$
\{\tau=n\} \in \mathcal{M}_{n} \cap \mathcal{E}(\Omega) \text { for all } n \in \mathbf{N} .
$$

For example, a constant stopping time i.e. $\tau=n_{0}$ for some $n_{0} \in \mathbf{N}$, is an $\mathcal{E}$-stopping time. And, for $A \in \mathcal{E}$, its first entry time $\tau_{A}$ and its first hitting time $\sigma_{A}$ are $\mathcal{E}$-stopping times ([11, Lemma 1.5]) :

$$
\begin{gathered}
\tau_{A}(\omega)=\inf \left\{n \in \mathbf{N} \mid X_{n}(\omega) \in A\right\} \quad \omega \in \Omega ; \\
\sigma_{A}(\omega)=\inf \left\{n \in \mathbf{N} \mid n \geq 1, X_{n}(\omega) \in A\right\} \quad \omega \in \Omega,
\end{gathered}
$$

where the infima of the empty set are understood to be $+\infty$.

In this paper, we deal with fuzzy sets in $\mathcal{G}(E)$ and we call maps from $\mathcal{G}(E)$ to $\mathcal{G}(E)$ fuzzy transition operators. We define a fuzzy transition operator $P: \mathcal{G}(E) \mapsto \mathcal{G}(E)$ by

$$
P \tilde{s}(x):=E_{x}\left(\tilde{s}\left(X_{1}\right)\right)=\sup _{y \in E}\{\tilde{q}(x, y) \wedge \tilde{s}(y)\} \quad x \in E \quad \text { for } \tilde{s} \in \mathcal{G}(E) .
$$

Next, we define fuzzy transition operators $P_{n}: \mathcal{G}(E) \mapsto \mathcal{G}(E)(n \in \mathbf{N})$ by

$$
P_{0}:=I(\text { identity }) \quad \text { and } \quad P_{n+1}:=P P_{n}(n=0,1,2, \cdots) .
$$

Then, we have

$$
P_{n} \tilde{s}(x):=E_{x}\left(\tilde{s}\left(X_{n}\right)\right) \quad x \in E \text { for } \tilde{s} \in \mathcal{G}(E) .
$$

Further, for an $\mathcal{E}$-stopping time $\tau$, we define a fuzzy transition operator $P_{\tau}: \mathcal{G}(E) \mapsto \mathcal{G}(E)$ by

$$
P_{\tau} \tilde{s}(x):=E_{x}\left(\tilde{s}\left(X_{\tau}\right)\right) \quad x \in E \quad \text { for } \tilde{s} \in \mathcal{G}(E),
$$

where we define $X_{\tau}:=X_{n}$ on $\{\tau=n\}, n \in \mathbf{N} \cup\{\infty\}$.

\section{Fuzzy transition operators}

In this section, we define various fuzzy transition operators and investigate the relations. For $A \in \mathcal{E}(E)$, we define a map $I_{A}: \mathcal{G}(E) \mapsto \mathcal{G}(E)$ by

$$
I_{A} \tilde{s}:=1_{A} \wedge \tilde{s} \text { for } \tilde{s} \in \mathcal{G}(E) \text {. }
$$

Then, the following lemma gives a decomposition of the hitting possibility $P_{\tau_{A}}$ by the fuzzy transition operator $P$.

Lemma 2.1. Let $A \in \mathcal{E}$ and $\tilde{s} \in \mathcal{G}(E)$. Then

$$
P_{\tau_{A}} \tilde{s}=\bigvee_{n \in \mathrm{N}}\left(I_{A^{c}} P\right)^{n} I_{A} \tilde{s} .
$$


Proof. From [11, Lemmas 1.1 and 3.1(i)], it is easily checked that $\left(I_{A^{c}} P\right)^{n} \tilde{s} \in \mathcal{G}(E)$ for $n \in \mathbf{N}$. By induction, we prove that for all $n \in \mathbf{N}$

$$
E_{x}\left(\tilde{s}\left(X_{\tau_{A}}\right) \wedge 1_{\left\{\tau_{A}=n\right\}}\right)=E_{x}\left(\tilde{s}\left(X_{n}\right) \wedge 1_{\left\{\tau_{A}=n\right\}}\right)=\left(I_{A^{c}} P\right)^{n} I_{A} \tilde{s}(x) \quad x \in E .
$$

When $n=0,(2.1)$ holds since

$$
E_{x}\left(\tilde{s}\left(X_{0}\right) \wedge 1_{\left\{\tau_{A}=0\right\}}\right)=1_{A}(x) \wedge \tilde{s}(x)=I_{A} \tilde{s}(x)=\left(I_{A^{c}} P\right)^{0} I_{A} \tilde{s}(x) \quad x \in E .
$$

Let $n \in \mathbf{N}$. We prove (2.1) for $n+1$, assuming that (2.1) holds for $n$. By using Markov property ([11, Theorem 2.2]) and the property of the fuzzy transition operator $P$, we obtain

$$
\begin{aligned}
\left(I_{A^{c}} P\right)^{n+1} I_{A} \tilde{s}(x) & =\left(I_{A^{c}} P\right)\left(I_{A^{c}} P\right)^{n} I_{A} \tilde{s}(x) \\
& =1_{A^{c}}(x) \wedge E_{x}\left(E_{X_{1}}\left(\tilde{s}\left(X_{n}\right) \wedge 1_{\left\{\tau_{A}=n\right\}}\right)\right) \\
& =1_{A^{c}}(x) \wedge E_{x}\left(\tilde{s}\left(X_{n+1}\right) \wedge 1_{\left\{\tau_{A} \circ \theta_{1}=n\right\}}\right) \\
& =E_{x}\left(\tilde{s}\left(X_{n+1}\right) \wedge 1_{\left\{\tau_{A}=n+1\right\}}\right) \quad x \in E .
\end{aligned}
$$

Thus, we get (2.1) inductively. We obtain this lemma from (2.1) and [11, Lemma 3.1(i)].

Next, we discuss the other relations among important fuzzy transition operators in dynamic fuzzy systems. Let $A \in \mathcal{E}$. Then, we define fuzzy transition operators on $\mathcal{G}(E)$ as follows :

$$
\begin{gathered}
G:=\bigvee_{n \in \mathrm{N}} P_{n} ; \quad G_{A}:=\bigvee_{n \in \mathrm{N}}\left(I_{A^{c}} P\right)^{n} ; \quad G_{A}^{\prime}:=\bigvee_{n \in \mathrm{N}}\left(P I_{A^{c}}\right)^{n} ; \\
U_{A}:=P G_{A}=G_{A}^{\prime} P=\bigvee_{n \in \mathrm{N}} P\left(I_{A^{c}} P\right)^{n}
\end{gathered}
$$

Then, clearly

$$
\begin{gathered}
G_{A}=I \vee I_{A^{c}} U_{A} ; \quad G_{A}^{\prime}=I \vee U_{A} I_{A^{c}} \\
G_{\phi}=G_{\phi}^{\prime}=G ; \quad G_{E}=G_{E}^{\prime}=I ; \quad U_{\phi}=P G ; \quad U_{E}=P .
\end{gathered}
$$

From Lemma 2.1, we also have

$$
G_{A} I_{A}=P_{\tau_{A}} ; \quad U_{A} I_{A}=P_{\sigma_{A}} .
$$

Further, we obtain the following resolvent equation (c.f. Revuz [9, Proposion 2.2.5]).

Theorem 2.1 (resolvent equation). Let $A, B \in \mathcal{E}$ satisfy $A \subset B$. Then:

$$
U_{A}=\bigvee_{n \in \mathrm{N}}\left(U_{B} I_{B \backslash A}\right)^{n} U_{B}=\bigvee_{n \in \mathrm{N}} U_{B}\left(I_{B \backslash A} U_{B}\right)^{n}
$$




$$
U_{A}=U_{B} \vee U_{B} I_{B \backslash A} U_{A}=U_{B} \vee U_{A} I_{B \backslash A} U_{B}
$$

Proof. Generally, for fuzzy transition operators $L, M, N: \mathcal{G}(E) \mapsto \mathcal{G}(E)$ and $n \in \mathbf{N}$, we can easily check

$$
(N(L \vee M))^{n} N=\bigvee_{\substack{k, n_{0}, n_{1}, \cdots, n_{k} \in \mathbb{N} \\ k+n_{0}+n_{1}+\cdots+n_{k}=n}}(N L)^{n_{0}} N M(N L)^{n_{1}} N M \cdots M(N L)^{n_{k}} N .
$$

Therefore,

$$
\bigvee_{n \in \mathbb{N}}(N(L \vee M))^{n} N=\bigvee_{k \in \mathbb{N}} \bigvee_{k, n_{0}, n_{1}, \cdots, n_{k} \in \mathbb{N}}\left((N L)^{n_{0}} N\right) M\left((N L)^{n_{1}} N\right) M \cdots M\left((N L)^{n_{k}} N\right)
$$

Putting $R:=\bigvee_{n \in \mathbb{N}}(N L)^{n} N$, we obtain

$$
\bigvee_{n \in \mathrm{N}}(N(L \vee M))^{n} N=\bigvee_{k \in \mathrm{N}}(R M)^{k} R .
$$

Let $A, B \in \mathcal{E}$ satisfy $A \subset B$. Here, taking $N:=P, L:=I_{B^{c}}, M:=I_{B \backslash A}$ in (2.8), we obtain $R=U_{B}$ and

$$
U_{A}=\bigvee_{n \in \mathbb{N}}\left(P I_{A^{c}}\right)^{n} P=\bigvee_{n \in \mathbb{N}}\left(P\left(I_{B^{c}} \vee I_{B \backslash A}\right)\right)^{n} P=\bigvee_{n \in \mathbb{N}}\left(U_{B} I_{B \backslash A}\right)^{n} U_{B}
$$

Therefore we obtain (2.6). We can easily check (2.7) from (2.6).

In the rest of this section, we prove the maximum principle for dynamic fuzzy systems (c.f. [9, Theorem 2.1.11]).

Lemma 2.2. Let $\tau$ be an $\mathcal{E}$-stopping time and $\tilde{s} \in \mathcal{G}(E)$. Then

$$
G \tilde{s}(x)=E_{x}\left(\bigvee_{n<\tau} \tilde{s}\left(X_{n}\right)\right) \vee P_{\tau} G \tilde{s}(x) \quad \text { for } x \in E .
$$

Proof. Using strong Markov property ([11, Theorem 2.2]), we have

$$
\begin{aligned}
P_{\tau} G \tilde{s}(x) & =E_{x}\left(G \tilde{s}\left(X_{\tau}\right)\right) \\
& =E_{x}\left(E_{X_{\tau}}\left(\bigvee_{n \geq 0} \tilde{s}\left(X_{n}\right)\right)\right) \\
& =E_{x}\left(\bigvee_{n \geq 0} \tilde{s}\left(X_{\tau+n}\right)\right) \\
& =E_{x}\left(\bigvee_{n \geq \tau} \tilde{s}\left(X_{n}\right)\right) \quad x \in E .
\end{aligned}
$$


Therefore we obtain this lemma.

We define a partial order $\leq$ on $\mathcal{G}(E)$ as follows : For $\tilde{s}, \tilde{r} \in \mathcal{G}(E)$,

$$
\tilde{s} \leq \tilde{r} \text { means that } \tilde{s}(x) \leq \tilde{r}(x) \text { for all } x \in E
$$

Theorem 2.2 (maximum principle). Let $\tilde{s}, \tilde{r} \in \mathcal{G}(E)$. Assume $\{\tilde{s}=0\} \in \mathcal{E}(E)$. If

$$
G \tilde{s} \leq G \tilde{r} \quad \text { on }\{\tilde{s}>0\} \text {, }
$$

then

$$
G \tilde{s} \leq G \tilde{r} \quad \text { on } E .
$$

Proof. First, we assume $\tilde{s} \in \mathcal{F}(E)$. Let $A:=\{\tilde{s}>0\}$. We have $A=\bigcup_{n=1}^{\infty}\{\tilde{s} \geq 1 / n\} \in$ $\mathcal{E}(E)$ from [11, Lemma 1.1]. We also have $A^{c}=\{\tilde{s}=0\} \in \mathcal{E}(E)$. Therefore $\tau_{A}$ is an $\mathcal{E}$-stopping time. From (2.9), we have

$$
P_{\tau_{A}} G \tilde{s} \leq P_{\tau_{A}} G \tilde{r}
$$

Then $E_{x}\left(\bigvee_{n<\tau_{A}}\left(\tilde{s}\left(X_{n}\right)\right)\right)=0$ for all $x \in E$, where we put $\bigvee_{n<0}\left(\tilde{s}\left(X_{n} \omega\right)\right):=0$ for $\omega \in \Omega$. Therefore, by (2.11) and Lemma 2.2, we obtain

$$
\begin{aligned}
G \tilde{s}(x) & =E_{x}\left(\bigvee_{n<\tau_{A}} \tilde{s}\left(X_{n}\right)\right) \vee P_{\tau_{A}} G \tilde{s}(x) \\
& \leq E_{x}\left(\bigvee_{n<\tau_{A}} \tilde{r}\left(X_{n}\right)\right) \vee P_{\tau_{A}} G \tilde{r}(x) \\
& =G \tilde{s}(x) \quad x \in E .
\end{aligned}
$$

Thus, we obtain (2.10) for $\tilde{s} \in \mathcal{F}(E)$. Next, we get the result for $\tilde{s} \in \mathcal{G}(E)$ by applying [11, Lemma 3.1(i)].

\section{3. $P$-excessive possibility measures}

First, we deal with possibility measures and introduce $P$-excessive property for them as a duality of the following $P$-superharmonic fuzzy sets in [11, Section 4$]$

Definition 3.1. A fuzzy set $\tilde{s} \in \mathcal{G}(E)$ is called $P$-superharmonic if

$$
\tilde{s}(x) \geq P \tilde{s}(x) \text { for all } x \in E \text {. }
$$

Definition $3.2([7,11])$. A map $\mu: \mathcal{E}(E) \mapsto[0,1]$ is called a possibility measure on $E$ if it satisfies (P.i) - (P.iii) :

(P.i) $\mu(\phi)=0$ and $\mu(E)=1$; 
(P.ii) If $A, B \in \mathcal{E}(E)$ satisfy $A \subset B$, then $\mu(A) \leq \mu(B)$;

(P.iii) If $\left\{A_{n}\right\}_{n=0}^{\infty} \subset \mathcal{E}(E)$, then $\mu\left(\bigcup_{n=0}^{\infty} A_{n}\right)=\bigvee_{n=0}^{\infty} \mu\left(A_{n}\right)$.

We define a fuzzy integration of a fuzzy set $\tilde{s} \in \mathcal{G}(E)$ by the possibility measure $\mu$ :

$$
\mu(\tilde{s}):=f_{E} \tilde{s}(x) \mathrm{d} \mu(x)=\sup _{\alpha \in[0,1]}\left(\alpha \wedge \mu\left(\tilde{s}_{\alpha}\right)\right) .
$$

Then, (P.i) - (P.iii) are equivalent to the following (I.i) - (I.iii) (see Ralescu and Adams [8]) :

(I.i) $\mu\left(1_{\phi}\right)=0$ and $\mu\left(1_{E}\right)=1$;

(I.ii) If $\tilde{s}, \tilde{r} \in \mathcal{G}(E)$ satisfy $\tilde{s} \leq \tilde{r}$, then $\mu(\tilde{s}) \leq \mu(\tilde{r})$;

(I.iii) If $\left\{\tilde{s}_{n}\right\}_{n=0}^{\infty} \subset \mathcal{G}(E)$, then $\mu\left(\bigvee_{n=0}^{\infty} \tilde{s}_{n}\right)=\bigvee_{n=0}^{\infty} \mu\left(\tilde{s}_{n}\right)$

Let $\mathcal{P}$ be the set of all possibility measures on $E$. We introduce a partial order on $\mathcal{P}$ as follows : For $\mu, \nu \in \mathcal{P}$,

$$
\mu \geq \nu \text { means that } \mu(A) \geq \nu(A) \text { for all } A \in \mathcal{E}(E) .
$$

By $[8],(3.1)$ is equivalent to

$$
\mu(\tilde{s}) \geq \nu(\tilde{s}) \text { for all } \tilde{s} \in \mathcal{G}(E) .
$$

We define $\mu L(\tilde{s}):=\mu(L \tilde{s})$ for $\tilde{s} \in \mathcal{G}(E)$ and fuzzy transition operators $L: \mathcal{G}(E) \mapsto \mathcal{G}(E)$.

Definition 3.3. A possibility measure $\mu \in \mathcal{P}$ is called $P$-excessive if

$$
\mu \geq \mu P
$$

We investigate properties of $P$-excessive possibility measures.

Lemma 3.1. Let $\mu \in \mathcal{P}$. $\mu$ is $P$-excessive if and only if

$$
\mu=\mu G \text {. }
$$

Proof. Let $\mu$ be $P$-excessive. Then

$$
\mu=\mu \vee \mu P \vee \mu P_{2} \vee \cdots \vee \mu P_{n} \quad \text { for all } n \in \mathbf{N}
$$

So we obtain (3.3). The converse proof is trivial.

We obtain the following lemma by Theorem 2.1 . 
Lemma 3.2 (c.f. $[9$, Proposition 2.2.6]). Let $\mu$ be P-excessive. Then :

$$
\mu I_{A} U_{A} \leq \mu I_{B} U_{B} \leq \mu \quad \text { for } A, B \in \mathcal{E}: A \subset B
$$

Proof. Let $A, B \in \mathcal{E}$ satisfy $A \subset B$. First we assume $\mu I_{B} U_{B} \leq \mu$. By induction, we prove

$$
\bigvee_{k=0}^{n-1} \mu I_{A} U_{B}\left(I_{B \backslash A} U_{B}\right)^{k} \vee \mu I_{B} U_{B}\left(I_{B \backslash A} U_{B}\right)^{n} \leq \mu I_{B} U_{B} \leq \mu \quad \text { for } n \in \mathbf{N}
$$

We have (3.5) for $n=1$ since

$$
\mu I_{A} U_{B} \vee \mu I_{B} U_{B} I_{B \backslash A} U_{B} \leq \mu I_{A} U_{B} \vee \mu I_{B \backslash A} U_{B} \leq \mu I_{B} U_{B} \leq \mu .
$$

Let $n \in \mathbf{N}$. We suppose (3.5) holds for $n$. Then, by multiplying of $I_{B \backslash A} U_{B}$ from the right side of (3.5) and taking its $\vee$-operation with $\mu I_{A} U_{B}$, we obtain

$$
\bigvee_{k=0}^{n} \mu I_{A} U_{B}\left(I_{B \backslash A} U_{B}\right)^{k} \vee \mu I_{B} U_{B}\left(I_{B \backslash A} U_{B}\right)^{n+1} \leq \mu I_{B \backslash A} U_{B} \vee \mu I_{A} U_{B}=\mu I_{B} U_{B} \leq \mu .
$$

Thus, we obtain (3.5) for all $n \in \mathbf{N}$. From (3.5), (2.6) and the property (I.iii) for $\mu$, we obtain

$$
\mu I_{A} U_{A}=\bigvee_{k=0}^{\infty} \mu I_{A} U_{B}\left(I_{B \backslash A} U_{B}\right)^{k} \leq \mu I_{B} U_{B} \leq \mu .
$$

Consequently, we get $\mu I_{A} U_{A} \leq \mu$ if $A, B \in \mathcal{E}(A \subset B)$ and $\mu I_{B} U_{B} \leq \mu$. Finally, since $\mu I_{E} U_{E}=\mu P \leq \mu$, the proof is completed.

Next, we give a duality for Snell's optimal stopping problem [11, Section 4]. The duality in Markov processes are found in [9, Propositions 2.5.6] and [2]. Let $\mu \in \mathcal{P}$ be a fixed initial possibility measure. Let $\tilde{s} \in \mathcal{G}(E)$ be a fuzzy goal. We consider a problem : Maximize $\mu P_{\tau}(\tilde{s})$ with respect to finite $\mathcal{E}$-stopping times. For simplicity, we deal with a case $\tilde{c}=1_{E}$ in [11, Section 4]. Then, we define a possibility measure

$$
\nu(\tilde{s}):=\sup _{\tau: \text { finite } \mathcal{E} \text {-stopping times }} \mu P_{\tau}(\tilde{s}), \quad \tilde{s} \in \mathcal{G}(E) .
$$

Lemma 3.3. $\nu$ has the following properties:

(i) $\nu=\mu G$;

(ii) $\nu=\mu \vee \nu P$.

Proof. (i) We have $\mu P_{\tau} \leq \mu G$ for all finite $\mathcal{E}$-stopping times $\tau$. Therefore $\nu \leq \mu G$. While, $\mu P_{n} \leq \nu$ for all $n \in \mathbf{N}$. By the property (I.iii) for $\mu$, we have $\nu \geq \mu G$. Therefore we obtain $\nu=\mu G$. 
(ii) From (i) and the property (I.iii), we obtain

$$
\nu=\mu G=\bigvee_{n \in \mathrm{N}} \mu P_{n}=\mu \vee \bigvee_{n \in \mathrm{N}} \mu P_{n} P=\mu \vee \mu G P=\mu \vee \nu P
$$

Therefore this lemma holds.

Theorem 3.1 (Duality in Snell's optimal stopping problem). The optimal stopping problem (3.6) has the following dual representation :

(i) $\nu$ is the smallest $P$-excessive possibility measure dominating $\mu$. Namely

$$
\nu(\tilde{s})=\min _{\mu^{\prime} \in \mathcal{P}: \mu^{\prime} \geq \mu, \mu^{\prime} \leq \mu^{\prime} P} \mu^{\prime}(\tilde{s}) \quad \text { for } \tilde{s} \in \mathcal{G}(E) .
$$

(ii) Further, it holds that

$$
\nu(\tilde{s})=\min _{\tilde{r}: P-\text { superharmonic, } \tilde{r}_{\geq} \tilde{s}} \mu(\tilde{r}) \quad \text { for } \tilde{s} \in \mathcal{G}(E) .
$$

Proof. (i) From Lemma 3.3(ii), we have $\nu \geq \mu$ and $\nu \geq \nu P$. Let $\mu^{\prime} \in \mathcal{P}$ be $P$-excessive such that $\mu^{\prime} \geq \mu$. Then, by Lemma 3.1,

$$
\mu^{\prime}(\tilde{s})=\mu^{\prime} G(\tilde{s})=\mu^{\prime}(G \tilde{s}) \geq \mu(G \tilde{s})=\mu G(\tilde{s})=\nu(\tilde{s}) \quad \text { for } \tilde{s} \in \mathcal{G}(E) .
$$

Therefore, we obtain (i).

(ii) From Lemma 3.3(ii) and [11, Lemma 4.1(ii)], we have

$\min \{\mu(\tilde{r}) \mid \tilde{r}$ is $P$-superharmonic and $\tilde{r} \geq \tilde{s}\}=\mu(G \tilde{s})=\mu G(\tilde{s})=\nu(\tilde{s}) \quad$ for $\tilde{s} \in \mathcal{G}(E)$.

Therefore, we obtain (ii) and the proof is completed.

Finally, by using Theorem 3.1, we obtain the following balayage theorem regarding $P$-excessive measures (c.f. $[9$, Theorem 2.4.5]).

Theorem 3.2 (co-balayage theorem). Let $\mu$ be $P$-excessive and let $A \in \mathcal{E}$. Then

$$
\mu H_{A}=\mu I_{A} G
$$

where $H_{A}:=I_{A} G_{A}^{\prime}=I_{A} \vee I_{A} U_{A} I_{A^{c}}$. Then, (3.10) is the smallest P-excessive possibility measure which dominates $\mu$ on $A$.

Proof. First, we prove that $\mu H_{A}$ is the smallest $P$-excessive possibility measure which dominates $\mu$ on $A$. We have

$$
\mu H_{A} I_{A}=\mu\left(I_{A} \vee I_{A} U_{A} I_{A^{c}}\right) I_{A}=\mu I_{A}
$$


Therefore $\mu H_{A}=\mu$ on $A$. Further, from (2.3) and Lemma 3.2, we have

$$
\mu H_{A} P=\mu I_{A} G_{A}^{\prime} P=\mu I_{A} U_{A}=\mu I_{A} U_{A} I_{A} \vee \mu I_{A} U_{A} I_{A^{c}} \leq \mu I_{A} \vee \mu I_{A} U_{A} I_{A^{c}}=\mu H_{A} .
$$

Thus $\mu H_{A}$ is $P$-excessive. While, let $\mu^{\prime} \in \mathcal{P}$ be $P$-excessive dominating $\mu$ on $A$. Using Lemma 3.2, we have

$$
\mu^{\prime}=\mu^{\prime} I_{A} \vee \mu^{\prime} I_{A^{c}} \geq \mu^{\prime} I_{A} \vee \mu^{\prime} I_{A} U_{A} I_{A^{c}} \geq \mu I_{A} \vee \mu I_{A} U_{A} I_{A^{c}}=\mu H_{A} .
$$

Thus, $\mu H_{A}$ is the smallest $P$-excessive dominating $\mu$ on $A$. Therefore, by Lemma 3.1(i) and Theorem 3.1(i), we obtain $\mu H_{A}=\mu I_{A} G$. Thus we obtain this theorem.

Corollary 3.1. Let $\mu$ be $P$-excessive. Then

$$
\mu I_{A} U_{A}=\mu I_{A} G P \quad \text { for } A \in \mathcal{E} .
$$

Proof. Trivial from Theorem 3.2 and (2.3).

\section{Dual dynamic fuzzy systems}

In this section, we deal with a dual system for dynamic fuzzy systems $X=\left\{X_{n}\right\}_{n \in \mathrm{N}}$ and we give an explicit representation for the duality in Theorem 3.1. We define a binary relation $\langle\cdot, \cdot\rangle$ on $\mathcal{G}(E) \times \mathcal{G}(E)$ :

$$
\langle\tilde{s}, \tilde{r}\rangle:=f_{E} \tilde{s}(x) \wedge \tilde{r}(x) \mathrm{d} x=\sup _{x \in E}\{\tilde{s}(x) \wedge \tilde{r}(x)\} \quad \text { for } \tilde{s}, \tilde{r} \in \mathcal{G}(E) .
$$

Define $\hat{q}(x, y):=\tilde{q}(y, x)$ for $x, y \in E$. Then, the fuzzy relation $\hat{q}$ is time-invariant and also satisfies the same normality condition. We may define a dual dynamic fuzzy system $\hat{X}=\left\{\hat{X}_{n}\right\}_{n \in \mathrm{N}}$ with a fuzzy transition operator $\hat{P}$ :

$$
\hat{P} \tilde{s}(x):=\sup _{y \in E}\{\hat{q}(x, y) \wedge \tilde{s}(y)\}=\sup _{y \in E}\{\tilde{s}(y) \wedge \tilde{q}(y, x)\} \quad x \in E \quad \text { for } \tilde{s} \in \mathcal{G}(E) .
$$

For the system, we use the notations " ' ' to distinguish $X$ and $\hat{X}$. The path space is $\hat{\Omega}:=$ $\Omega$ and a path is $\hat{\omega}=(\hat{\omega}(0), \hat{\omega}(1), \hat{\omega}(2), \cdots) \in \hat{\Omega}$. We define $\hat{X}_{n}(\hat{\omega}):=\hat{\omega}(n)(\hat{\omega} \in \hat{\Omega}, n \in \mathbf{N})$ and we put $\sigma$-fields by $\hat{\mathcal{M}}_{n}:=\sigma\left(\hat{X}_{0}, \hat{X}_{1}, \cdots, \hat{X}_{n}\right)(n \in \mathbf{N})$ and $\hat{\mathcal{M}}:=\sigma\left(\bigcup_{n \in \mathbb{N}} \hat{\mathcal{M}}_{n}\right)$. Then, we also define a possibility measure $\hat{P}(\Lambda):=\sup _{\hat{\omega} \in \Lambda} \bigwedge_{n=0}^{\infty} \hat{q}\left(\hat{X}_{n}(\hat{\omega}), \hat{X}_{n+1}(\hat{\omega})\right)$ for $\Lambda \in \hat{\mathcal{M}}$. For $h \in \mathcal{F}(\hat{\mathcal{M}})$, we define an expectation by the possibility measure $\hat{P}$ :

$$
\hat{E}_{x}(h):=f_{\{\hat{\omega} \in \hat{\Omega}: \hat{\omega}(0)=x\}} h(\hat{\omega}) \mathrm{d} \hat{P}(\hat{\omega})=\sup _{\hat{\omega} \in \hat{\Omega}: \hat{\omega}(0)=x} h(\hat{\omega}) \wedge \bigwedge_{n=0}^{\infty} \hat{q}\left(\hat{X}_{n} \hat{\omega}, \hat{X}_{n+1} \hat{\omega}\right) \quad \text { for } x \in E .
$$

Then, we have the following lemma.

Lemma 4.1. Let $\tilde{s}, \tilde{r} \in \mathcal{G}(E)$ and $\left\{\tilde{s}_{n}\right\}_{n=0}^{\infty} \subset \mathcal{G}(E)$. Then : 
(i) $\langle P \tilde{s}, \tilde{r}\rangle=\langle\tilde{s}, \hat{P} \tilde{r}\rangle$;

(ii) $\left\langle I_{A} \tilde{s}, \tilde{r}\right\rangle=\left\langle\tilde{s}, I_{A} \tilde{r}\right\rangle \quad$ for $A \in \mathcal{E}(E)$;

(iii) $\langle\tilde{s}, \tilde{r}\rangle=\langle\tilde{r}, \tilde{s}\rangle$;

(iv) $\langle\tilde{s}, \tilde{s}\rangle=\langle\tilde{s}, 1\rangle=\sup _{x \in E} \tilde{s}(x)$;

(v) $\langle 0, \tilde{s}\rangle=\langle\tilde{s}, 0\rangle=0$;

(vi) $\left\langle\bigvee_{n \geq 0} \tilde{s}_{n}, \tilde{r}\right\rangle=\sup _{n \geq 0}\left\langle\tilde{s}_{n}, \tilde{r}\right\rangle$.

Proof. (i) We have

$$
\left.\langle P \tilde{s}, \tilde{r}\rangle=\sup _{x \in E}\left\{\left\{\sup _{y \in E} \tilde{q}(x, y) \wedge \tilde{s}(y)\right\} \wedge \tilde{r}(x)\right\}=\sup _{y \in E} \tilde{s}(y) \wedge\left\{\sup _{x \in E} \tilde{r}(x) \wedge \tilde{q}(x, y)\right\}\right\}=\langle\tilde{s}, \hat{P} \tilde{r}\rangle .
$$

(ii) - (vi) are trivial from the definitions.

Define the first entry time $\hat{\tau}_{A}$ and the first hitting time $\hat{\sigma}_{A}$ for $A \in \mathcal{E}$ for the dual system $\hat{X}$ similarly to Section 1 , and define possibilities $\hat{P}_{\hat{\tau}_{A}}$ and $\hat{P}_{\hat{\sigma}_{A}}$ similarly to Section 2. Then we have the following results.

Proposition 4.1. Let $\tilde{s}, \tilde{r} \in \mathcal{G}(E)$ and $A \in \mathcal{E}$. Then :

(i) $\left\langle U_{A} \tilde{s}, \tilde{r}\right\rangle=\left\langle\tilde{s}, \hat{U}_{A} \tilde{r}\right\rangle$, where $\hat{U}_{A}:=\bigvee_{n \in \mathrm{N}} \hat{P}\left(I_{A^{c}} \hat{P}\right)^{n}$;

(ii) $\langle G \tilde{s}, \tilde{r}\rangle=\langle\tilde{s}, \hat{G} \tilde{r}\rangle$, where $\hat{G}:=\bigvee_{n \in \mathrm{N}} \hat{P}_{n}$;

(iii) $\left\langle H_{A} \tilde{s}, \tilde{r}\right\rangle=\left\langle\tilde{s}, \hat{P}_{\hat{\tau}_{A}} \tilde{r}\right\rangle$;

(iv) $\left\langle I_{A} U_{A} \tilde{s}, \tilde{r}\right\rangle=\left\langle\tilde{s}, \hat{P}_{\hat{\sigma}_{A}} \tilde{r}\right\rangle$;

(v) $\left\langle I_{A} U_{A} I_{A} \tilde{s}, \tilde{r}\right\rangle=\left\langle\tilde{s}, I_{A} \hat{U}_{A} I_{A} \tilde{r}\right\rangle$.

Proof. (i) From (2.3) and Lemma 4.1, we have

$$
\left\langle U_{A} \tilde{s}, \tilde{r}\right\rangle=\left\langle\bigvee_{n \in \mathbf{N}}\left(P I_{A^{c}}\right)^{n} P \tilde{s}, \tilde{r}\right\rangle=\sup _{n \in \mathbf{N}}\left\langle\left(P I_{A^{c}}\right)^{n} P \tilde{s}, \tilde{r}\right\rangle=\sup _{n \in \mathbf{N}}\left\langle\tilde{s}, \hat{P}\left(I_{A^{c}} \hat{P}\right)^{n} \tilde{r}\right\rangle=\left\langle\tilde{s}, \hat{U}_{A} \tilde{r}\right\rangle
$$

(ii) and (v) are similar. (iii) By (2.3) and Lemma 4.1(ii)(vi), similarly to (i) we have

$$
\left\langle H_{A} \tilde{s}, \tilde{r}\right\rangle=\left\langle I_{A} \tilde{s}, \tilde{r}\right\rangle \vee\left\langle I_{A} U_{A} I_{A^{c}} \tilde{s}, \tilde{r}\right\rangle=\left\langle\tilde{s}, I_{A} \tilde{r}\right\rangle \vee\left\langle\tilde{s}, I_{A^{c}} \hat{U}_{A} I_{A} \tilde{r}\right\rangle=\left\langle\tilde{s}, \bigvee_{n \in \mathrm{N}}\left(I_{A^{c}} \hat{P}\right)^{n} I_{A} \tilde{r}\right\rangle=\left\langle\tilde{s}, \hat{P}_{\hat{\tau}_{A}} \tilde{r}\right\rangle
$$

Thus we obtain (iii). Finally, (iv) follows from

$$
\bigvee_{n \in \mathrm{N}}\left(I_{A^{c}} \hat{P}\right)^{n} I_{A} \tilde{r}=\hat{P}_{\hat{\tau}_{A}} \tilde{r} \quad \text { for } \tilde{r} \in \mathcal{G}(E)
$$


Therefore we obtain this proposition.

The following lemma characterizes densities of $P$-excessive possibility measures.

Lemma 4.2. Let $\tilde{s} \in \mathcal{G}(E)$ satisfy $\sup _{x \in E} \tilde{s}(x)=1$. Put $\mu \in \mathcal{P}$ by

$$
\mu(A):= \begin{cases}\sup _{x \in A} \tilde{s}(x) & \text { for } A \in \mathcal{E}(E)(A \neq \emptyset), \\ 0 & \text { for } A=\emptyset .\end{cases}
$$

Then, the possibility measure $\mu$ is $P$-excessive if and only if $\tilde{s}$ is $\hat{P}$-superharmonic.

Proof. Suppose that $\tilde{s}$ is $\hat{P}$-superharmonic. Then we have

$$
\begin{aligned}
\mu P(A) & =\mu\left(P 1_{A}\right) \\
& =\sup _{x \in E}\left\{\tilde{s}(x) \wedge P 1_{A}(x)\right\} \\
& =\sup _{x \in E}\left\{\tilde{s}(x) \wedge \sup _{y \in A} \tilde{q}(x, y)\right\} \\
& =\sup _{y \in A} \sup _{x \in E}\{\tilde{s}(x) \wedge \tilde{q}(x, y)\} \\
& =\sup _{y \in A} \hat{P} \tilde{s}(y) \\
& \leq \sup _{y \in A} \tilde{s}(y) \\
& =\mu(A), \quad A \in \mathcal{E}(E) .
\end{aligned}
$$

Therefore we have $\mu$ is $P$-excessive. Conversely, if $\mu$ is $P$-excessive, then similarly we obtain

$$
\sup _{y \in A} \hat{P} \tilde{s}(y) \leq \sup _{y \in A} \tilde{s}(y), \quad A \in \mathcal{E}(E) .
$$

Taking $A:=\{y\}(y \in E)$, we have that $\tilde{s}$ is $\hat{P}$-superharmonic. Therefore, the proof is completed.

Next, we investigate a possibility measure $\xi$ relative to which the dynamic fuzzy systems $X$ and $\hat{X}$ are dual. Let $\tilde{m} \in \mathcal{G}(E)$ satisfy $\sup _{x \in E} \tilde{m}(x)=1$. Let $\xi$ be a fixed possibility measure with the density $\tilde{m}$ :

$$
\xi(A):= \begin{cases}\sup _{x \in A} \tilde{m}(x) & \text { for } A \in \mathcal{E}(E)(A \neq \emptyset), \\ 0 & \text { for } A=\emptyset .\end{cases}
$$

We define a binary relation $\langle\cdot, \cdot\rangle_{\xi}$ on $\mathcal{G}(E) \times \mathcal{G}(E)$ :

$$
\langle\tilde{s}, \tilde{r}\rangle_{\xi}:=f_{E} \tilde{s}(x) \wedge \tilde{r}(x) \mathrm{d} \xi(x)=\sup _{x \in E}\{\tilde{s}(x) \wedge \tilde{r}(x) \wedge \tilde{m}(x)\} \quad \text { for } \tilde{s}, \tilde{r} \in \mathcal{G}(E) .
$$

Theorem 4.1. The dynamic fuzzy systems $X$ and $\hat{X}$ are dual relative to the possibility measure $\xi$ if and only if $\xi$ is $P$-excessive and $\hat{P}$-excessive. Then, for $\tilde{s}, \tilde{r} \in \mathcal{G}(E)$ and $\left\{\tilde{s}_{n}\right\}_{n=0}^{\infty} \subset \mathcal{G}(E)$, we have : 
(i) $\langle P \tilde{s}, \tilde{r}\rangle_{\xi}=\langle\tilde{s}, \hat{P} \tilde{r}\rangle_{\xi}$;

(ii) $\left\langle I_{A} \tilde{s}, \tilde{r}\right\rangle_{\xi}=\left\langle\tilde{s}, I_{A} \tilde{r}\right\rangle_{\xi} \quad$ for $A \in \mathcal{E}(E)$;

(iii) $\langle\tilde{s}, \tilde{r}\rangle_{\xi}=\langle\tilde{r}, \tilde{s}\rangle_{\xi}$;

(iv) $\langle\tilde{s}, \tilde{s}\rangle_{\xi}=\langle\tilde{s}, 1\rangle_{\xi}=\sup _{x \in E}\{\tilde{s}(x) \wedge \tilde{m}(x)\}$

(v) $\langle 0, \tilde{s}\rangle_{\xi}=\langle\tilde{s}, 0\rangle_{\xi}=0$;

(vi) $\left\langle\bigvee_{n \geq 0} \tilde{s}_{n}, \tilde{r}\right\rangle_{\xi}=\sup _{n \geq 0}\left\langle\tilde{s}_{n}, \tilde{r}\right\rangle_{\xi}$;

(vii) $\left\langle U_{A} \tilde{s}, \tilde{r}\right\rangle_{\xi}=\left\langle\tilde{s}, \hat{U}_{A} \tilde{r}\right\rangle_{\xi}$, where $\hat{U}_{A}:=\bigvee_{n \in \mathrm{N}} \hat{P}\left(I_{A^{c}} \hat{P}\right)^{n}$.

(viii) $\langle G \tilde{s}, \tilde{r}\rangle_{\xi}=\langle\tilde{s}, \hat{G} \tilde{r}\rangle_{\xi}$, where $\hat{G}:=\bigvee_{n \in \mathrm{N}} \hat{P}_{n}$;

(ix) $\left\langle H_{A} \tilde{s}, \tilde{r}\right\rangle_{\xi}=\left\langle\tilde{s}, \hat{P}_{\hat{\tau}_{A}} \tilde{r}\right\rangle_{\xi}$;

(x) $\left\langle I_{A} U_{A} \tilde{s}, \tilde{r}\right\rangle_{\xi}=\left\langle\tilde{s}, \hat{P}_{\hat{\sigma}_{A}} \tilde{r}\right\rangle_{\xi}$

(xi) $\left\langle I_{A} U_{A} I_{A} \tilde{s}, \tilde{r}\right\rangle_{\xi}=\left\langle\tilde{s}, I_{A} \hat{U}_{A} I_{A} \tilde{r}\right\rangle_{\xi}$.

Proof. If $\xi$ is $P$-excessive, then we have

$$
\begin{aligned}
\tilde{m}(y) & =\mu\left(1_{\{y\}}\right) \\
& \geq \mu\left(P 1_{\{y\}}\right) \\
& =\mu\left(\sup _{y^{\prime} \in E}\left\{\tilde{q}\left(\cdot, y^{\prime}\right) \wedge 1_{\{y\}}\left(y^{\prime}\right)\right\}\right) \\
& =\mu(\tilde{q}(\cdot, y)) \\
& =\sup _{x \in E}\{\tilde{q}(x, y) \wedge \tilde{m}(x)\} \\
& \geq \tilde{q}(x, y) \wedge \tilde{m}(x) \quad \text { for all } x, y \in E .
\end{aligned}
$$

Therefore,

$$
\tilde{q}(x, y) \wedge \tilde{m}(x) \leq \tilde{q}(x, y) \wedge \tilde{m}(y) \text { for all } x, y \in E .
$$

Further, if $\xi$ is $\hat{P}$-excessive, in the same way, we have the reverse inequality in (4.5). Therefore

$$
\tilde{q}(x, y) \wedge \tilde{m}(x)=\tilde{q}(x, y) \wedge \tilde{m}(y) \text { for all } x, y \in E .
$$

Similarly, we can easily derive that $\xi$ is $P$-excessive and $\hat{P}$-excessive from (4.6). Therefore (4.6) is equivalent to the condition that $\xi$ is $P$-excessive and $\hat{P}$-excessive. While, from (4.6), we obtain

$$
\begin{aligned}
\langle P \tilde{s}, \tilde{r}\rangle_{\xi} & =\sup _{x \in E}\left\{\left\{\sup _{y \in E} \tilde{q}(x, y) \wedge \tilde{s}(y)\right\} \wedge \tilde{r}(x) \wedge \tilde{m}(x)\right\} \\
& =\sup _{y \in E}\left\{\tilde{s}(y) \wedge\left\{\sup _{x \in E} \tilde{r}(x) \wedge \tilde{q}(x, y)\right\} \wedge \tilde{m}(x)\right\} \\
& =\langle\tilde{s}, \hat{P} \tilde{r}\rangle_{\xi} .
\end{aligned}
$$


Conversely, in (4.7) we take $\tilde{s}:=1_{\{y\}}$ and $\tilde{r}:=1_{\{x\}}$ for $x, y \in E$. Then (4.7) is reduced to (4.6). Therefore, $X$ and $\hat{X}$ are dual relative to the possibility measure $\xi$ if and only if $\xi$ is $P$-excessive and $\hat{P}$-excessive. The proof of (i) - (xi) are similar to the proofs of Lemma 4.1 and Proposition 4.1.

In the rest of this section, we discuss Snell's optimal stopping problem in the duality of dynamic fuzzy systems. We use the binary relation $\langle\cdot, \cdot\rangle$ on $\mathcal{G}(E) \times \mathcal{G}(E)$. In the problem (3.6), we consider a case when an initial possibility measure $\mu$ is represented by

$$
\mu(A):=\sup _{x \in A} \tilde{u}(x), \quad A \in \mathcal{E}(E) .
$$

Then, from (3.6), we have

$$
\nu(\tilde{s}):=\sup _{\tau: \text { finite } \mathcal{E}-\text { stopping times }}\left\langle\tilde{u}, P_{\tau} \tilde{s}\right\rangle, \quad \tilde{s} \in \mathcal{G}(E) .
$$

Theorem 4.2. The optimal stopping problem (4.9) has the following dual representation:

$$
\nu(\tilde{s})=\min _{\tilde{v}: \hat{P} \text {-superharmonic, } \tilde{v} \geq \tilde{u}}\langle\tilde{v}, \tilde{s}\rangle=\min _{\tilde{r}: P \text {-superharmonic, } \tilde{r} \geq \tilde{s}}\langle\tilde{u}, \tilde{r}\rangle .
$$

Proof. From (4.8) and (3.8), it is trivial that

$$
\nu(\tilde{s})=\min _{\tilde{r}: P-\text { superharmonic }, \tilde{r} \geq \tilde{s}}\langle\tilde{u}, \tilde{r}\rangle .
$$

It is sufficient to prove that

$$
\nu(\tilde{s})=\min _{\tilde{v}: \hat{P} \text {-superharmonic, } \tilde{v} \geq \tilde{u}}\langle\tilde{v}, \tilde{s}\rangle .
$$

Let $\tilde{v}$ be $\hat{P}$-superharmonic and $\tilde{v} \geq \tilde{u}$, and put a possibility measure $\mu^{\prime}$ by

$$
\mu^{\prime}(A):=\sup _{x \in A} \tilde{v}(x), \quad A \in \mathcal{E}(E) .
$$

From Lemma 4.2, $\mu^{\prime}$ is $P$-excessive and $\mu^{\prime} \geq \mu$. By (3.7), we obtain

$$
\nu(\tilde{s}) \leq \mu^{\prime}(\tilde{s})=\langle\tilde{v}, \tilde{s}\rangle .
$$

Especially, $\hat{G} \tilde{u}$ is $\hat{P}$-superharmonic and $\hat{G} \tilde{u} \geq \tilde{u}$ since

$$
\hat{G} \tilde{u}=\bigvee_{n \geq 0} \hat{P}^{n} \tilde{u}=\tilde{u} \bigvee \bigvee_{n \geq 1} \hat{P}^{n} \tilde{u}=\tilde{u} \vee \hat{P} \hat{G} \tilde{u}
$$

Then, from (3.9) and Proposition 4.1(ii), we obtain

$$
\nu(\tilde{s})=\mu G(\tilde{s})=\mu(G \tilde{s})=\langle\tilde{u}, G \tilde{s}\rangle=\langle\hat{G} \tilde{u}, \tilde{s}\rangle
$$


Together with (4.13), we obtain (4.12). Therefore, the proof is completed.

\section{A numerical example}

We consider a numerical example with a one-dimensional state space $E=\mathbf{R}$, where $\mathbf{R}$ is the set of all real numbers. We calculate the optimal value $\nu(\tilde{s})$ for Snell's problem (4.9).

Example 5.1. We give fuzzy sets $\tilde{s}, \tilde{u}$ and a fuzzy relation $\tilde{q}$ by

$$
\begin{gathered}
\tilde{s}(x)=(0.8-0.1|1-x|) \vee 0, \quad x \in \mathbf{R}, \\
\tilde{u}(x)=(0.8-0.1|1+x|) \vee 0, \quad x \in \mathbf{R}, \\
\tilde{q}(x, y)=\left(1-\left|y-x^{3}\right|\right) \vee 0, \quad x, y \in \mathbf{R} .
\end{gathered}
$$

then, we give a possibility measure $\mu$ by (4.8). Let $\hat{s}^{*}$ be the smallest $\hat{P}$-superharmonic fuzzy set dominating $\tilde{s}$. By [13, Example 4.1], we have

$$
\tilde{s}^{*}(x)= \begin{cases}\tilde{s}(x) & \text { for } x \in\left(-\infty, x_{1}\right) \approx(-\infty,-1.1651) \\ \tilde{q}(x, x) & \text { for } x \in\left[x_{1}, x_{2}\right) \approx[-1.1651,-1.1147) \\ \alpha^{*} \approx 0.7296 & \text { for } x \in\left[x_{2}, x_{3}\right) \approx[-1.1147,0.2964) \\ \tilde{s}(x) & \text { for } x \in\left[x_{3}, x_{4}\right) \approx[0.2964,0.8687) \\ \tilde{q}(x, x) & \text { for } x \in\left[x_{4}, x_{5}\right) \approx[0.8687,0.8789) \\ 0.8 & \text { for } x \in\left[x_{5}, x_{6}\right) \approx[0.8789,1.0880) \\ \tilde{q}(x, x) & \text { for } x \in\left[x_{6}, x_{7}\right) \approx[1.0880,1.0916) \\ \tilde{s}(x) & \text { for } x \in\left[x_{7},+\infty\right) \approx[1.0916,+\infty) .\end{cases}
$$

Next, let $\tilde{u}^{*}$ be the smallest $\hat{P}$-superharmonic fuzzy set dominating $\tilde{u}$. Since $\hat{q}(x, y)=$ $\tilde{q}(y, x)(x, y \in \mathbf{R})$, in a method similar to [13, Example 4.1], we can easily check

$$
\tilde{u}^{*}(x)=\left\{\begin{array}{lll}
0.8 & \text { for } x \in\left(-\infty, x_{8}\right) & \approx(-\infty, 0.2091) \\
\tilde{q}(x, x) & \text { for } x \in\left[x_{8}, x_{9}\right) & \approx[0.2091,0.4098) \\
\tilde{u}(x) & \text { for } x \in\left[x_{9}, x_{10}\right) & \approx[0.4098,0.6749) \\
0.6325 & \text { for } x \in\left[x_{10},+\infty\right) & \approx[0.6749,+\infty) .
\end{array}\right.
$$

Fig. 5.1 shows the fuzzy sets $\tilde{s}$ and $\tilde{u}^{*}$, and Fig. 5.2 shows the fuzzy sets $\tilde{u}$ and $\hat{s}^{*}$. 


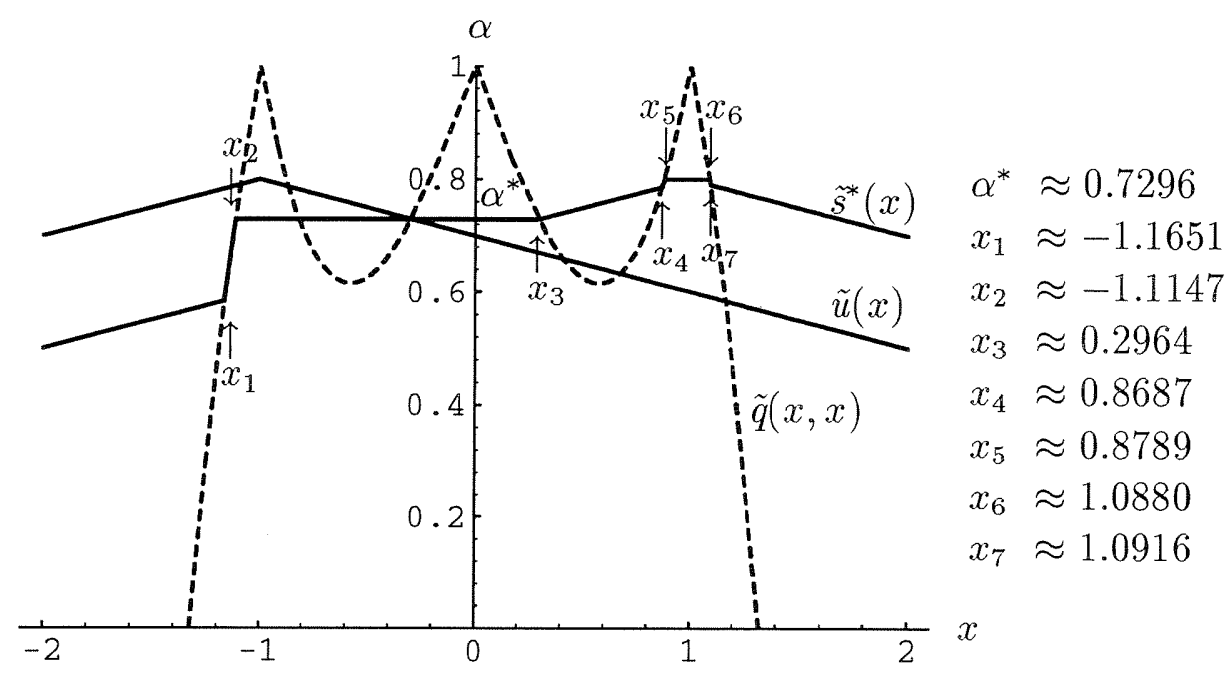

Fig. 5.1. The fuzzy sets $\tilde{s}(x)$ and $\tilde{u}^{*}(x)$ and a fuzzy set $\tilde{q}(x, x)$.

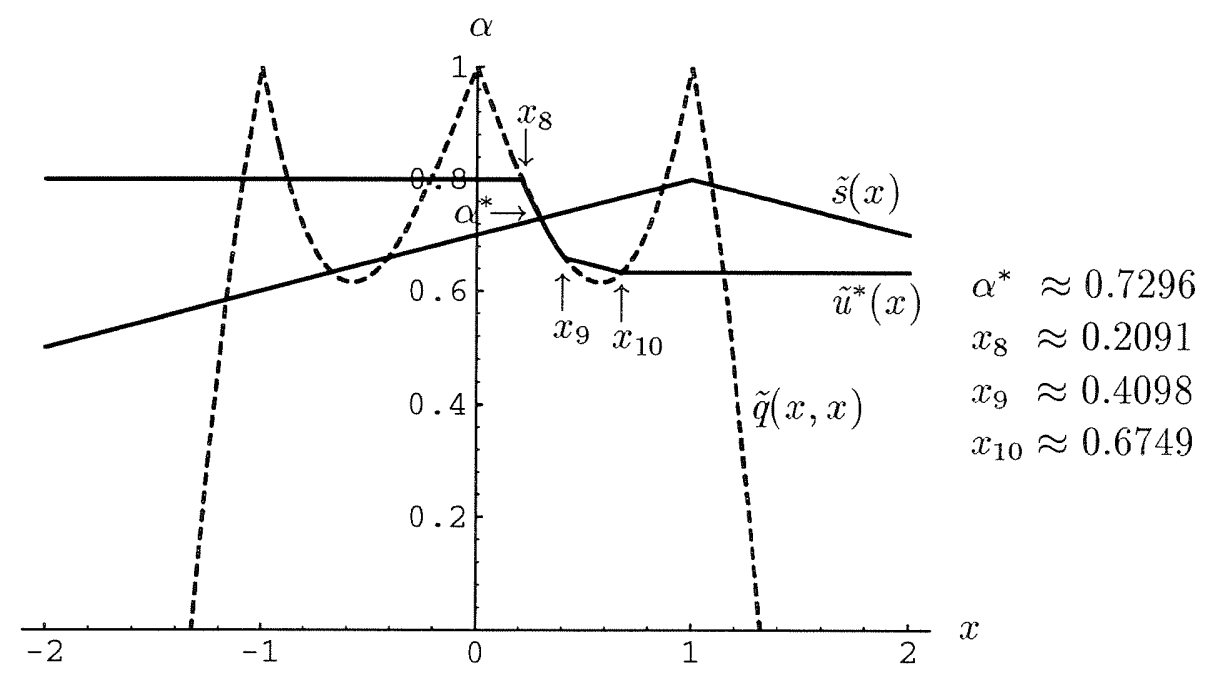

Fig. 5.2. The fuzzy sets $\tilde{u}(x)$ and $\tilde{s}^{*}(x)$ and a fuzzy set $\tilde{q}(x, x)$.

From Theorem 4.2 and Figs. 5.1 and 5.2, we obtain

$$
\nu(\tilde{s})=\left\langle\tilde{u}^{*}, \tilde{s}\right\rangle=\left\langle\tilde{u}, \hat{s}^{*}\right\rangle=\alpha^{*} \approx 0.7296 .
$$

\section{References}

[1] B.:E.Bellman and L.A.Zadeh, Decision-making in a fuzzy environment, Management Sci. Ser B. 17 (1970) 141-164.

[2] Bismut, J.-M., Sur un Problème de Dynkin, Z. Wahr. Verw. Geb. 39 (1977) 31-53.

[3] K.L.Chung, Markov Chains with Stationary Transition Probabilities (Springer, Berlin, 1960). 
[4] M.Kurano, M.Yasuda, J.Nakagami and Y.Yoshida, A limit theorem in some dynamic fuzzy systems, Fuzzy Sets and Systems 51 (1992) 83-88.

[5] K.Kuratowski, Topology I (Academic Press, New York, 1966).

[6] V.Novàk, Fuzzy Sets and Their Applications (Adam Hilder, Bristol-Boston, 1989).

[7] M.L.Puri and D.Ralescu, A possibility measure is not a fuzzy measure, Fuzzy Sets and Systems 29 (1982) 311-312.

[8] D.Ralescu and Adams, The fuzzy integral, J. Math. Anal. Appl. 75 (1980) 562-570.

[9] D.Revuz, Markov Chains, the second edition (North-Holland, New York, 1984).

[10] M.Sugeno, Fuzzy measures and fuzzy integral : a survey in M.M.Gupta, G.N.Saridis and B.R.Gaines, Eds., Fuzzy Automata and Decision Processes (North-Holland, Amsterdam, 1977) 89-102.

[11] Y.Yoshida, Markov chains with a transition possibility measure and fuzzy dynamic programming, Fuzzy Sets and Systems 66 (1994) 39-57.

[12] Y.Yoshida, The recurrence of dynamic fuzzy systems, RIFIS Technical Report 80 (1994), Research Institute of Fundamental Information Science, Kyushu University, Japan.

[13] Y.Yoshida, Superharmonic fuzzy sets on recurrent sets in dynamic fuzzy systems, submitted.

[14] Y.Yoshida, M.Yasuda, J.Nakagami and M.Kurano, A potential of fuzzy relations with a linear structure: The contractive case, Fuzzy Sets and Systems 60 (1993) 283-294.

[15] Y.Yoshida, M.Yasuda, J.Nakagami and M.Kurano, A potential of fuzzy relations with a linear structure: The unbounded case, Fuzzy Sets and Systems 66 (1994) 83-95.

[16] L.A.Zadeh, Fuzzy sets, Inform. and Control 8 (1965) 338-353. 